DOI: https://doi.org/10.14311/TPFM.2021.002

\title{
SECOND-ORDER MODEL FOR ATMOSPHERIC TURBULENCE WITHOUT CRITICAL RICHARDSON NUMBER
}

\author{
Matteo Caggio ${ }^{1}$, Mario Schiavon ${ }^{2,3}$, Francesco Tampieri ${ }^{3}$, Tomáš Bodnár ${ }^{4,5}$, \\ ${ }^{1}$ Department of Mathematics, Faculty of Science, University of Zagreb, Croatia. \\ 2 Department of Physics and Astronomy, University of Bologna, Bologna, Italy. \\ ${ }^{3}$ National Research Council of Italy, Institute of Atmospheric Sciences and Climate (CNR- \\ ISAC), Bologna, Italy. \\ ${ }^{4}$ Institute of Mathematics, Czech Academy of Sciences, Žitná 25, 11567 Prague 1, Czech \\ Republic. \\ ${ }^{5}$ Faculty of Mechanical Engineering, Czech Technical University in Prague, Karlovo Náměstí \\ 13, 12135 Prague 2, Czech Republic.
}

In honor of José Manuel Redondo

\begin{abstract}
Abstract: The purpose of this communication is to present a derivation of the non-dimensional vertical gradients of the mean wind speed and mean potential temperature expressed in terms of the so-called similarity functions for very stable conditions of the atmosphere where theoretical approaches provide conflicting results (see e.g. Luhar et al. [19]). The result is based on the analysis of the second-order model equations in the boundary layer approximations in which new heat flux equations are proposed. The model employs a recent closure for the pressure-temperature correlation, avoiding the issue of a critical treshold for the Richardson number.
\end{abstract}

Keywords: Atmospheric boundary layer, second-order closure model, turbulence parameterisations, strong stratification, critical Richardson number.

\section{Introduction}

In the last decades, higher-order turbulence closure models in atmospheric turbulence have been largely successful (see Mellor and Yamada [22]; Launder et al. [17]; Lumley [20]; André et al. [1]; Mellor and Yamada [23]; Galperin et al. [8]; Canuto [4]; Shih and Shabbir [28]; Nakanishi [25]; Cheng et al. [6]; Kantha and Clayson [13], [14]; Sukoriansky et al. [30]; Galperin et al. [9]; Canuto et al. [5]; Kantha and Carniel [15]; Bretherton and Park [2]; Zilitinkevich et al. [33]). These models are derived from the basic dynamic equations of motion through the use of suitable turbulence closures. One of the most popular model of turbulence have been proposed by Mellor and Yamada [23]; hereafter MY82. In spite of the wide use of this model, it presents limitations recently discussed by some authors. One of them is the presence of a critical treshold for the Richardson number, beyond which turbulence decays. The predicted value, $\mathrm{Ri}_{c r} \approx 0.19$, is too small since various data show that turbulence does exist for $\mathrm{Ri}>>1$. In the years, several authors re-examined the MY82 model and its variants addressing its deficiencies (see e.g. Galperin et al. [8], Cheng et al. [6], Canuto et al. [5], Kantha and Carniel [15], Zilitinkevich et al. [33]). For example, the model proposed by Cheng et al. [6] increases $\mathrm{Ri}_{c r}$ to $O(1)$, while the Canuto et al. [5] model does not have a finite $\mathrm{Ri}_{c r}$. Further comparison among MY82 and models without critical Richardson number can be found in Li et al. [18]. Their modifications resulted in an improved agreement with observational, experimental, and high-resolution numerical data. However, an inconvenience of these models lies in a more complex structure than MY82 and none as efficient and convenient as a previous modification due to Galperin et al. [8], whose formulation simplifies the original MY82 model while preserving its physics. As mentioned in Cheng et al. [7], the model of Galperin et al. [8] was welcomed by the geophysics community and became widely used. 
However, the limitation related to the critical treshold of the Richardson number is still present in their model. Very recently, Cheng et al. [7] introduced a second-order turbulence closure model with new horizontal and vertical heat flux equations and without a critical treshold for the Richardson number, consistent with meteorological observation, laboratory experimental, direct numerical simulation (DNS) and large-eddy simulation (LES) data. The authors mentioned that their model is structurally simpler than the Galperin et al. [8] model. The novelty of the model lies in a new closure for the return-to-isotropy term of the pressure-temperature correlation. This closure was previously proposed by Canuto et al. [5] when they examined the wavenumber spectra of the pressure-temperature relaxation time scale [see Eq. (9c) in Canuto et al. [5]].

In the following, we consider the new model proposed by Cheng et al. [7] and by using the analysis developed by Caggio and Bodnár [3] together with the analysis developed by Cheng et al. [7], we compute the non-dimensional vertical gradients of the mean wind speed and mean potential temperature expressed in terms of the so-called similarity functions for conditions ranging from neutrality to very large stability. A comparison with previous results will be also discussed.

\section{Second-order scheme}

We introduce the second-order turbulent equations in the boundary layer approximation. The model below is derived from the second-order equations for the turbulent variables in which suitable closure assumptions are considered, under horizontally homogeneous conditions, neglecting timetendency, advection and diffusion terms (for more details see the Appendix below). The system of equations reads as follows ${ }^{1}$

$$
\begin{gathered}
\overline{u^{2}}=\gamma_{1} q^{2}+2 C_{2} \frac{l_{1}}{q} \beta g \overline{w \theta}-6 \frac{l_{1}}{q} \overline{u w} \frac{\partial U}{\partial z}, \overline{v^{2}}=\gamma_{1} q^{2}+2 C_{2} \frac{l_{1}}{q} \beta g \overline{w \theta}, \overline{w^{2}}=\gamma_{1} q^{2}+2\left(3-2 C_{2}\right) \frac{l_{1}}{q} \beta g \overline{w \theta} \\
\overline{u w}=3 \frac{l_{1}}{q}\left[-\left(\overline{w^{2}}-C_{1} q^{2}\right) \frac{\partial U}{\partial z}+\left(1-C_{2}\right) \beta g \overline{u \theta}\right] \\
\overline{u \theta}=3 \frac{l_{2}}{q}\left[-\overline{u w} \frac{\partial \Theta}{\partial z}-\left(1-C_{4}\right) \overline{w \theta} \frac{\partial U}{\partial z}\right], \overline{w \theta}=3 \frac{l_{2}}{q}\left[-\overline{w^{2}} \frac{\partial \Theta}{\partial z}+\left(1-C_{3}\right) \beta g \overline{\theta^{2}}\right] \\
\overline{\theta^{2}}=-\frac{\Lambda_{2}}{q} \overline{w \theta} \frac{\partial \Theta}{\partial z}, \frac{q^{3}}{\Lambda_{1}}=\left(-\overline{u w} \frac{\partial U}{\partial z}+\beta g \overline{w \theta}\right)
\end{gathered}
$$

where, without loss of generality, we oriented our coordinate system so that $\overline{v w}=0$ and $\overline{u w}$ is aligned with the mean wind vector $U$ in the $x$-direction. Moreover, it may be shown (see Mellor [21]) that $\partial V / \partial z=\overline{u v}=\overline{v \theta}=0$. Here, $\gamma_{1}=\frac{1}{3}-2 \frac{A_{1}}{B_{1}}$ and $\left(l_{1}, l_{2}, \Lambda_{1}, \Lambda_{2}\right)=\left(A_{1}, A_{2}, B_{1}, B_{2}\right) l$, where $A_{1}, A_{2}, B_{1}$ and $B_{2}$ are positive coefficients and $l$ represents a length scale such that $l \rightarrow \kappa z$ as $z \rightarrow 0$ where $\kappa$ is the von Kármán constant. This scale can be prescribed or solved from a prognostic equation. Later, we will introduce the length scale proposed by Cheng et al. [7].

The second equation in (4) is the budget for the turbulent kinetic energy under equilibrium conditions, namely production of turbulence by shear and buoyancy is totally balanced by the dissipation of turbulence. This is physically acceptable in the surface-layer, but not necessarily outside of it. Note that we dropped the diffusion contribution under the assumptions (possibly incorrect both for stable and unstable conditions) that the second-order terms are constant with the height (see Mellor [21]).

The above system is presented in the framework of the MY82 model. The closure proposed by Cheng et al. [7] results in the following modification

$$
\frac{l_{2}}{l}=\frac{A_{2}^{\prime}}{\left(1+\sigma_{t}\right)}, \quad A_{2}^{\prime}=\left(1+\sigma_{t 0}\right),
$$

\footnotetext{
${ }^{1}$ Here and hereafter, capital and tiny letters are meant as mean physical quantity and its turbulent fluctuation respectively in the sense of Reynolds decomposition. Overbar denotes ensemble average. In this sense we split the wind speed in $U+u$ and the potential temperature in $\Theta+\theta$. Quantities like $\overline{u w}, \overline{w \theta}$ or analogous, are interpreted as turbulent stress and heat fluxes, respectively.
} 
where $\sigma_{t}$ is the turbulent Prandtl number defined as

$$
\sigma_{t}=\frac{-P_{s} \mathrm{Ri}}{P_{b}}
$$

with $\sigma_{t 0}$ its neutral value and

$$
P_{s}=-\overline{u w} \frac{\partial U}{\partial z}, \quad P_{b}=\beta g \overline{w \theta}
$$

are the production terms due to shear and buoyancy respectively. Here, Ri is the gradient Richardson number defined as

$$
\mathrm{Ri}=\frac{N^{2}}{S^{2}}, \quad N^{2}=\beta g \frac{\partial \Theta}{\partial z}, \quad S^{2}=\left(\frac{\partial U}{\partial z}\right)^{2} .
$$

Positive values of $\mathrm{Ri}$ imply stable conditions of the atmosphere, negative values unstable conditions. With the modification (5), $\overline{u \theta}$ and $\overline{w \theta}$ in (3) change as follows (see Appendix A in Cheng et al. [7] for a more detailed derivation of the new heat flux equations)

$$
\overline{u \theta}=-3 \frac{A_{2}^{\prime} l}{q}\left[\left(1-C_{4}\right) \overline{w \theta} \frac{\partial U}{\partial z}\right], \quad \overline{w \theta}=-3 \frac{A_{2}^{\prime} l}{q}\left[\overline{w^{2}} \frac{\partial \Theta}{\partial z}-\left(1-C_{3}\right) \beta g \overline{\theta^{2}}\right]-\left(\overline{u w} \frac{\partial \Theta}{\partial z}\right) / \frac{\partial U}{\partial z} .
$$

Consequently, in the following analysis we will consider the system (1), (2), (4), (9) with values of the coefficients from Cheng et al. [7], namely

$$
A_{1}=0.92, \quad A_{2}^{\prime}=1.332, \quad B_{1}=16.6, \quad B_{2}=10.1, \quad C_{1}=0.08, \quad C_{2}=0.25, \quad C_{3}=0.22, \quad C_{4}=0 .
$$

\subsection{Stability and similarity functions}

In the framework of Mellor and Yamada [23], we express the turbulent stress $\overline{u w}$ and the heat flux $\overline{w \theta}$ in terms of the common (although possibly incorrect) flux-gradient approximation

$$
\overline{u w}=-K_{m} \frac{\partial U}{\partial z}=-l q S_{m} \frac{\partial U}{\partial z}, \overline{w \theta}=-K_{h} \frac{\partial \Theta}{\partial z}=-l q S_{h} \frac{\partial \Theta}{\partial z},
$$

where $K_{m}$ and $K_{h}$ are the eddy diffusivities, functions of $l, q$ and non-dimensional stability functions $S_{m}$ and $S_{h}$. Moreover, we define (see Mellor and Yamada [23])

$$
G_{m}=\frac{l^{2}}{q^{2}}\left(\frac{\partial U}{\partial z}\right)^{2}, G_{h}=-\frac{l^{2}}{q^{2}} \frac{g}{\theta_{0}} \frac{\partial \Theta}{\partial z} .
$$

Using (11) and (12) we can rewrite the turbulent kinetic energy budget equation (second equation in (4)) as follows

$$
\frac{P_{s}+P_{b}}{\varepsilon}=B_{1}\left(S_{m} G_{m}+S_{h} G_{h}\right)
$$

where $\varepsilon=q^{3} / \Lambda_{1}$ is the turbulent kinetic energy dissipation. Equilibrium conditions require $\left(P_{s}+\right.$ $\left.P_{b}\right) / \varepsilon=1$ and we end up with

$$
B_{1}\left(S_{m} G_{m}+S_{h} G_{h}\right)=1 .
$$

Now, from (1), (2) (4), (9), (11) and (12) we can derive the stability functions $S_{m}$ and $S_{h}$ in terms of the gradient Richardson number, namely

$$
S_{h}=C /\left[1-G_{h}(A+B)\right]
$$

and

$$
S_{m}=\left[c^{\prime}-\left(c^{\prime}(A+B)-C\left(a^{\prime}+b^{\prime}\right)\right) G_{h}\right] /\left[1-G_{h}(A+B)\right]
$$

where

$$
\begin{gathered}
A=a-a^{\prime}, \quad B=b-b^{\prime}, \quad C=c-c^{\prime} \\
a^{\prime}=9 A_{1} A_{2}^{\prime}\left(1-C_{2}\right)\left(1-C_{4}\right), \quad b^{\prime}=6 A_{1}^{2}\left(3-2 C_{2}\right), \quad c^{\prime}=3 A_{1}\left(\gamma_{1}-C_{1}\right)
\end{gathered}
$$




$$
a=3 A_{2}^{\prime} B_{2}\left(1-C_{3}\right), \quad b=6 A_{1} A_{2}^{\prime}\left(3-2 C_{2}\right), \quad c=3 A_{2}^{\prime} \gamma_{1}
$$

and $G_{h}$ solution of the second-order equation

$$
\begin{gathered}
c_{2} G_{h}^{2}+c_{1} G_{h}+c_{0}=0, \\
c_{0}=-\mathrm{Ri}, \quad c_{1}=\left(B_{1} s_{0}+d_{1}\right) \mathrm{Ri}-B_{1} s_{2}, \quad c_{2}=B_{1} s_{3}
\end{gathered}
$$

with

$$
s_{0}=C, \quad s_{2}=c^{\prime}, \quad s_{3}=c^{\prime}(A+B)-C\left(a^{\prime}+b^{\prime}\right), \quad d_{1}=A+B .
$$

Now, in the framework of the Monin-Obukhov Similarity Theory (see Monin and Obukhov [24]) we define the non-dimensional vertical gradients of the mean wind speed $U$ and mean potential temperature $\Theta$ as follows:

$$
\frac{\kappa z}{u_{*}} \frac{\partial U}{\partial z}=\phi_{m}\left(\frac{z}{L}\right), \frac{\kappa z u_{*}}{H} \frac{\partial \Theta}{\partial z}=-\frac{\kappa z}{\theta_{*}} \frac{\partial \Theta}{\partial z} \equiv \phi_{h}\left(\frac{z}{L}\right)
$$

where $u_{*}^{2}=\overline{u w}, H=-\overline{w \theta}, \theta_{*}=-H / u_{*}$ and $L=u_{*}^{3} \theta_{0} / \kappa g H$ is a length scale first introduced by Obukhov [26] that can be interpreted as a measure of the stability. The sign of the heat flux determines the sign of $L$, negative for unstable cases $(\overline{w \theta}>0, H<0)$, positive for stable cases $(\overline{w \theta}<0, H>0)$ (see for example Garratt [10], Tampieri [31] and Wyngaard [32]). In the following, we are interested in the behavior of $\phi_{m}$ that could be expressed as (see Cheng et al. [7])

$$
\phi_{m}=\frac{G_{m}^{1 / 4}}{S_{m}^{1 / 2}} \frac{\kappa z}{l}
$$

In terms of the gradient Richardson number, $G_{m}$ can be derived from the turbulent kinetic energy balance (13) with $G_{h}$ from (16). Moreover, in Eq. (18) the length scale $l$ must be parameterized. In near neutral conditions it can be assumed to be proportional to the distance from the surface $z$. A common extension to stable conditions is

$$
l=\kappa z /(1+\widetilde{\alpha} z / L), \quad \widetilde{\alpha}=2.7,
$$

with a limitation on the stability range $0 \leq z / L<1$ (see Cheng et al. [7], relation (11e)). To exploit the behaviour of the present theory as the stability range is extended we refer again to Nakanishi [25], relation (39), who suggest a limit for $l$ that shall not become smaller that the value at $z / L=1$. Thus, the following formula is used in order to interpolate between the different parameterizations: ${ }^{2}$

$$
\frac{\kappa z}{l}=\frac{\widetilde{\beta}\left(1+\alpha^{\prime} \frac{z}{L}\right)}{\widetilde{\beta}+\alpha^{\prime} \frac{z}{L}}=\frac{\widetilde{\beta}\left(1+\alpha^{\prime} \phi_{m} \operatorname{Ri} \frac{S_{h}}{S_{m}}\right)}{\widetilde{\beta}+\alpha^{\prime} \phi_{m} \operatorname{Ri} \frac{S_{h}}{S_{m}}}, \quad\left(\alpha^{\prime}=\frac{\widetilde{\alpha}}{1-\frac{1}{\widetilde{\beta}}}, \widetilde{\alpha}=2.7, \widetilde{\beta}=3.7\right) .
$$

Consequently, we can derive $\phi_{m}$ solving the following relation

$$
b_{2} \phi_{m}^{2}+b_{1} \phi_{m}+b_{0}=0
$$

where

$$
b_{2}=\alpha^{\prime} \operatorname{Ri} \frac{S_{h}}{S_{m}}, \quad b_{1}=\widetilde{\beta}\left(1-\alpha^{\prime} \operatorname{Ri} \frac{S_{h}}{S_{m}} \frac{G_{m}^{1 / 4}}{S_{m}^{1 / 2}}\right), \quad b_{0}=-\widetilde{\beta} \frac{G_{m}^{1 / 4}}{S_{m}^{1 / 2}} .
$$

\footnotetext{
${ }^{2}$ The relation (20) could be derived introducing another stability parameter, the flux Richardson number $R_{f}=$ $\frac{g}{\theta_{0}} \overline{w \theta} / \overline{u w} \frac{\partial U}{\partial z}=\frac{z}{L} \phi_{m}^{-1}$, for which, in analogy with $L$, positive values imply stable conditions of the atmosphere, and noticing that, using relations (11) we can express $R_{f}$ in terms of Ri and the stability functions $S_{m}$ and $S_{h}$ as follows: $R_{f}=\operatorname{Ri}\left(S_{h} / S_{m}\right)$. The empirical values of the coefficients $\widetilde{\alpha}, \widetilde{\beta}$ are from Nakanishi [25].
} 


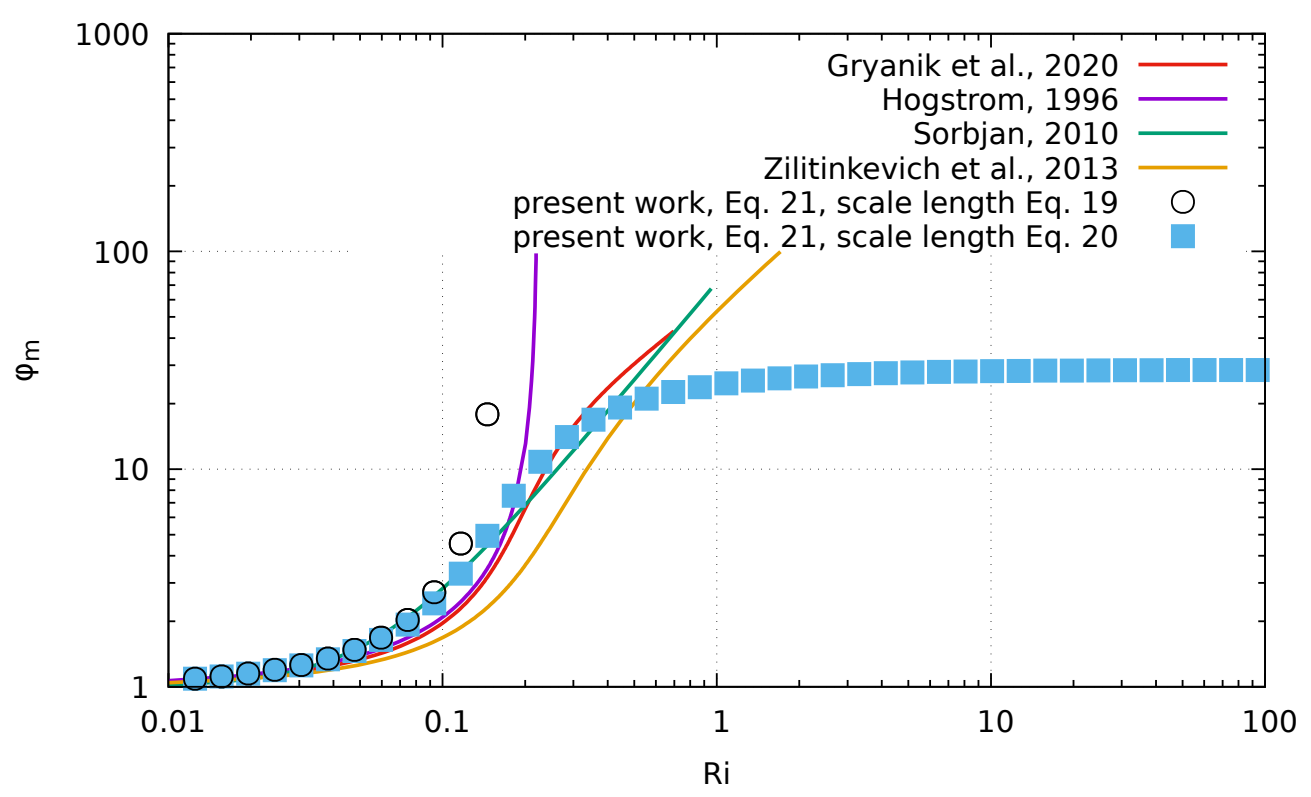

Figure 1: Similarity function $\phi_{m}$ as function of Ri. Blue squares: similarity function from Eq. (21) with the scale length parameterized as in Eq. (20). Open dots: the same, with the scale length from Eq. (19). Coloured continuous lines: expression from various authors (see text for some details).

\section{Results and conclusions}

The similarity function $\phi_{m}$ computed from Eq. (21) with parameterization (20) is reported in Fig. 1 (full squares) and compared with some literature results. At first, it results that our solution exists for the range $R i>0$, that is, it does not present any treshold. This solution is compared with the commonly used log-linear similarity functions for velocity and temperature from Högström [12] (violet), that shows a critical Richardson number, and with the functions suggested by Sorbjan [29] (green) and by Gryanik et al. [11], based on observations, and thus limited in the Ri range. The theoretical curve by Zilitinkevich et al. [33] (yellow) based on a different closure also do not exhibit a critical Richardson number. It results that in the near neutral and weak stability range all the solutions are equivalent; while they become quite different as stability increases. It is also interesting to note that the present approach, if the scale length is allowed to decrease without limit, as in Eq. (19), exhibits a singular behaviour at a finite value of $R i$, and thus does not give a solution for larger $R i$. This behaviour is shown in the figure (open dots) and highligths the key role of the lenght scale parameterization.

\section{Appendix}

In this Appendix we first introduce the general second-order equations for the turbulent variables. Then, we discuss the parameterisation made in order to close the system and obtain the equations (1) - (4). The discussion below follows exactly the same line as in Caggio and Bodnár (2018).

The second-order equations for turbulent variables reads as follows (see e.g. Garrat [10], Tampieri [31], Wyngaard [32])

$$
\begin{gathered}
\frac{\partial \overline{u_{i} u_{j}}}{\partial t}+\frac{\partial}{\partial x_{k}}\left[U_{k} \overline{u_{i} u_{j}}+\overline{u_{k} u_{i} u_{j}}-\nu \frac{\partial}{\partial x_{k}} \overline{u_{i} u_{j}}\right]+\frac{1}{\rho_{0}}\left(\frac{\partial}{\partial x_{j}} \overline{p u_{i}}+\frac{\partial}{\partial x_{i}} \overline{p u_{j}}\right) \\
=-\overline{u_{k} u_{i}} \frac{\partial U_{j}}{\partial x_{k}}-\overline{u_{k} u_{j}} \frac{\partial U_{i}}{\partial x_{k}}-\frac{1}{\theta_{0}}\left(g_{j} \overline{u_{i} \theta}+g_{i} \overline{u_{j} \theta}\right)+\frac{1}{\rho_{0}} \overline{p\left(\frac{\partial u_{i}}{\partial x_{j}}+\frac{\partial u_{j}}{\partial x_{i}}\right)}-2 \nu \overline{\frac{\partial u_{i}}{\partial x_{k}} \frac{\partial u_{j}}{\partial x_{k}}}
\end{gathered}
$$




$$
\begin{aligned}
& \frac{\partial \overline{u_{j} \theta}}{\partial t}+\frac{\partial}{\partial x_{k}}\left[U_{k} \overline{\theta u_{j}}+\overline{u_{k} u_{j} \theta}-\alpha \overline{u_{j} \frac{\partial \theta}{\partial x_{k}}}-\nu \overline{\frac{\partial u_{j}}{\partial x_{k}}}\right]+\frac{1}{\rho_{0}}\left(\frac{\partial}{\partial x_{j}} \overline{p \theta}\right) \\
= & -\overline{u_{j} u_{k}} \frac{\partial \Theta}{\partial x_{k}}-\overline{\theta u_{k}} \frac{\partial U_{j}}{\partial x_{k}}-\frac{1}{\theta_{0}} g_{j} \overline{\theta^{2}}+\frac{1}{\rho_{0}}\left(p \overline{\frac{\partial \theta}{\partial x_{j}}}\right)-(\alpha+\nu) \overline{\frac{\partial u_{j}}{\partial x_{k}} \frac{\partial \theta}{\partial x_{k}}}, \\
& \frac{\partial \overline{\theta^{2}}}{\partial t}+\frac{\partial}{\partial x_{k}}\left[U_{k} \overline{\theta^{2}}+\overline{u_{k} \theta^{2}}-\alpha \frac{\partial \overline{\theta^{2}}}{\partial x_{k}}\right]=-2 \overline{u_{k} \theta} \frac{\partial \Theta}{\partial x_{k}}-2 \alpha \frac{\partial \theta}{\partial x_{k}} \frac{\partial \theta}{\partial x_{k}},
\end{aligned}
$$

where $g_{j}=(0,0,-g)$ is the gravity acceleration, $\beta=1 / \theta_{0}, \nu$ is the kinematic viscosity and $\alpha$ the thermal diffusivity. The Coriolis parameter has been neglected as a standard simplification in the surface-layer.

In order to close the system (22) - (24), we express the third-order terms as (see e.g Nakanishi $[25])$ :

$$
\begin{gathered}
\overline{p\left(\frac{\partial u_{i}}{\partial x_{j}}+\frac{\partial u_{j}}{\partial x_{i}}\right)}=-\frac{q}{3 l_{1}}\left(\overline{u_{i} u_{j}}-\frac{\delta_{i j}}{3} q^{2}\right)+C_{1} q^{2}\left(\frac{\partial U_{i}}{\partial x_{j}}+\frac{\partial U_{j}}{\partial x_{i}}\right)+C_{2} \beta\left(g_{i} \overline{u_{j} \theta}+g_{j} \overline{u_{i} \theta}-\frac{2}{3} \delta_{i j} g_{k} \overline{u_{k} \theta}\right), \\
\overline{p \frac{\partial \theta}{\partial x_{j}}}=-\frac{q}{3 l_{2}} \overline{u_{j} \theta}+C_{3} \beta g_{j} \overline{\theta^{2}}+C_{4} \overline{u_{k} \theta} \frac{\partial U_{i}}{\partial x_{k}} \\
\overline{u_{k} u_{i} u_{j}}=-q \lambda_{1}\left(\frac{\partial \overline{u_{i} u_{j}}}{\partial x_{k}}+\frac{\partial \overline{u_{i} u_{k}}}{\partial x_{j}}+\frac{\partial \overline{u_{j} u_{k}}}{\partial x_{i}}\right), \overline{u_{k} u_{j} \theta}=-q \lambda_{2}\left(\frac{\partial \overline{u_{k} \theta}}{\partial x_{j}}+\frac{\partial \overline{u_{j} \theta}}{\partial x_{k}}\right) \\
\overline{u_{k} \theta^{2}}=-q \lambda_{3} \frac{\partial \overline{\theta^{2}}}{\partial x_{k}}
\end{gathered}
$$

The terms containing the pressure have been closed in the sense of return-to-isotropy proposed by Rotta [27], namely an anisotropic flow tends to isotropy in absence of external forcings.

The terms containing third-order covariances of velocity components and temperature are expressed in terms of the flux-gradient approximation. According to the Kolmogorov hypothesis of small-scale isotropy (see Kolmogorov [16]), the dissipative terms are expressed as

$$
2 \nu \overline{\frac{\partial u_{i}}{\partial x_{k}} \frac{\partial u_{j}}{\partial x_{k}}}=\frac{2}{3} \frac{q^{3}}{\Lambda_{1}} \delta_{i j}, \quad 2 \alpha \overline{\frac{\partial \theta}{\partial x_{k}} \frac{\partial \theta}{\partial x_{k}}}=2 \frac{q}{\Lambda_{2}} \overline{\theta^{2}} .
$$

Moreover, we have (see e.g. Mellor [21])

$$
(\alpha+\nu) \overline{\frac{\partial u_{j}}{\partial x_{k}} \frac{\partial \theta}{\partial x_{k}}}=0,
$$

since there is no isotropic first-order tensor. Here, $l_{1}, l_{2}, \lambda_{1}, \lambda_{2}, \lambda_{3}$ and $\Lambda_{1}, \Lambda_{2}$ are length scales and $C_{1}, C_{2}, C_{3}$ and $C_{4}$ are positive coefficients. The quantity $q$ represents the square-root of the turbulent kinetic energy. Finally, pressure diffusional terms are small in the first place (see e.g. Mellor [21]),

$$
\overline{p u_{i}}=\overline{p \theta}=0 .
$$

\section{Acknowledgment}

M. C. has been fully supported by the Croatian Science Foundation under the project MultiFM IP-2019-04-1140. T. B. was supported by the Czech Science Foundation under the grant No. P201-19-04243S. 


\section{References}

[1] André J. C., G. DeMoor, P. Lacarrére and R. du Vachat, 1978: Modeling the 24-hour evolution of the mean and turbulent structures of the planetary boundary layer, J. Atmos. Sci., $\mathbf{3 5}$, 1861-1883.

[2] Bretherton C. S. and S. Park, 2009: A new moist turbulence parameterization in the Community Atmosphere Model, J. Climate, 22, 3422-3448.

[3] Caggio M. and T. Bodnár 2018: Analysis of the Turbulence Parameterisations for the Atmospheric Surface Layer, In Proceedings Topical Problems of Fluid Mechanics 2018, Prague, 2018 Edited by David Šimurda and Tomáš Bodnár, pp. 31-38.

[4] Canuto V. M., 1992: Turbulent convection with overshooting: Reynolds stress approach, Astrophys. J., 392, 218-232.

[5] Canuto V. M., Y. Cheng, A. M. Howard and E. N. Essau, 2008: Stably stratified flows: A model with no $\operatorname{Ri}(\mathrm{cr})$, , 65, 2437-2447.

[6] Cheng Y., V. M. Canuto and A. M. Howard, 2002: An improved model for the turbulent PBL, J. Atmos. Sci., 59, 1550-1565.

[7] Cheng Y., V. M. Canuto, A. M. Howard, A. S. Ackerman, M. Kelley, A. M. Fridlind, G. A. Schmidt, M. S. Yao, A. Del Genio and G. S. Elsaesser, 2020: A Second-Order Closure Turbulence Model: New Heat Flux Equations and No Critical Richardson Number, J. Atmos. Sci., $77(8), 2743-2759$.

[8] Galperin B., L. H. Kantha, S. Hassid and A. Rosati, 1988: A quasi-equilibrium turbulent energy model for geophysical flows, J. Atmos. Sci., 45, 55-62.

[9] Galperin B., S. Sukoriannsky and P. S. Anderson, 2007: On the critical Richardson number in stably stratified turbulence Atmos. Sci. Lett., 8, 65-69.

[10] Garratt J. R., 1992: The atmospheric boundary layer, Cambridge University Press.

[11] Gryanik V. M., C. Lüpkes, A. Grachev and D. Sydorenko, 2020: New Modified and Extended Stability Functions for the Stable Boundary Layer based on SHEBA and Parametrizations of Bulk Transfer Coefficients for Climate Models, J. Atmos. Sci., 77(8), 2687-2716

[12] Högström U., 1988: Non-dimensional wind and temperature profiles in the atmospheric surface layer: A re-evaluation, Bound.-Layer Meteor., 42, 55-78.

[13] Kantha L. H., and C. A. Clayson, 1994: An improved mixed layer model for geophysical applications, J. Geophys. Res., 99, 25 235-25 266.

[14] Kantha L. H., and C. A. Clayson, 2004: On the effect of surface gravity waves on mixing in the oceanic mixed layer, Ocean Modell., 6, 101-124.

[15] Kantha L. H., and S. Carniel, 2009: A note on modeling mixing in stably stratified flows, J. Atmos. Sci., 66, 2501-2505.

[16] Kolmogorov A. N., 1941a: The local structure of turbulence in incompressible viscous fluid for very large Reynolds numbers, Dokl. Akad. Nauk SSSR, 30, 299-303; for English translation see Selected works of A. N. Kolmogorov, I, ed. V.M. Tikhomirov, pp. 321-318, Kluwer, 1991.

[17] Launder B. E., G. Reece and W. Rodi, 1975: Progress in the development of a Reynolds-stress turbulent closure, J. Fluid Mech., 68, 537-566.

[18] Li D., G. G. Katul and S. S. Zilitinkevich, 2016: Closure schemes for stably stratified atmospheric flows whitout turbulence cutoff, J. Atmos. Sci., 73, 4817-4832. 
[19] Luhar A. K., P. J. Hurley and K. N. Rayner, 2009: Modelling near-surface low winds over land under stable conditions: sensitivity tests, flux-gradient relationships, and stability parameters, Bound. Layer Meteorol., 130, 249-274.

[20] Lumley J. L., 1979: Computational modeling of turbulent flows, Adv. Appl. Mech., 18, 123176.

[21] Mellor G. L., 1973: Analytic prediction of the properties of stratified planetary surface layer, J. Atmos. Sci., 30, 1061-1069.

[22] Mellor G. L. and T. Yamada, 1974: A hierarchy of turbulence closure models for planetary boundary layers, J. Atmos. Sci.., 31, 1791-1806.

[23] Mellor G. L. and T. Yamada, 1982: Development of a turbulence closure model for geophysical fluid problems, Rev. Geophys. Space Phys., 20, 851-875.

[24] Monin A. S. and A. M. Obukhov, 1954: Osnovnye zakonomernosti turbulentnogo peremeshivanija $\mathrm{v}$ prizemnomsloe atmosfery (Basic laws of turbulent mixing in the atmosphere near the ground), Trudy geofiz. inst. AN SSSR, 24(151), 163-187.

[25] Nakanishi M., 2001: Improvement of the Mellor - Yamada turbulence closure model based on large eddy simulation data, Bound. Layer Meteor., 99, 349-378.

[26] Obukhov A. M., 1946: Turbulence in thermally inhomogeneous atmosphere, Trudy In-ta Theoret. Geofiz. AN SSSR, 1, 95-115. In Russian.

[27] Rotta J. C., 1951: Statisthe Theorie nichthomogener Turbulenz. Z. Phys., 129, 547-572.

[28] Shih T. -H. and A. Shabbir, 1992: Advances in modeling the pressure correlation terms in the second moment equations, Studies in Turbulence, T. B. Gatski, S. Sarkar, and C. G. Speziale, Eds., Springer, 91-128.

[29] Sorbjan Z. and A. A. Grachev, 2010: An Evaluation of the Flux-Gradient Relationship in the Stable Boundary Layer, Bound. Layer Meteor., 135, 385-405.

[30] Sukoriansky S., B. Galperin and I. Staroselsky, 2005: A quasinormal scale elimination model of turbulent flows with stable stratification, Phys. Fluids, 17, 085107.

[31] Tampieri F., 2017: Turbulence and Dispersion in the Planetary Boundary Layer, Physics of Earth and Space Environments, Springer International Publishing.

[32] Wyngaard J. C., 2010: Turbulence in the Atmosphere, Cambridge University Press.

[33] Zilitinkevich S. S., T. Elperin, N. Kleeorin, I. Rogachevskii and I. N. Esau, 2013: A hierarchy of energy- and flux-budget (EFB) turbulence closure models for stably-stratified geophysical flows, Bound. Layer Meteor., 146, 341-373. 